- Original Article

\title{
Predictors of Successful Smoking Cessation after Inpatient Intervention for Stroke Patients
}

\author{
Eugene Ha, Jun-Yong Jo, Ah-Leum Ahn*, Eun-Jung Oh, Jae-Kyung Choi, Dong-Yung Cho, Hyuk-Jung Kweon \\ Department of Family Medicine, Konkuk University Medical Center, Seoul, Korea
}

Background: Smoking is a well-known risk factor of cancer, chronic disease, and cerebrovascular disease. Hospital admission is a good time to quit smoking but patients have little opportunity to take part in an intensive smoking cessation intervention. The purpose of this study was to identify the factors of successful smoking cessation among stroke patients who undergo an intensive cessation intervention during the hospitalization period.

Methods: Thirty-nine male smokers who were admitted with stroke were enrolled in the study. They participated in a smoking cessation intervention during hospitalization. Smoking status was followed up by telephone 3 months later. Nicotine dependence, sociodemographic factors, and other clinical characteristics were assessed.

Results: After 3 months post-intervention, the number of patients who stopped smoking was 27 (69.2\%). In addition, there was no significant difference in nicotine dependence, sociodemographic factors, and clinical characteristics. Only the stages of readiness for smoking cessation were a significant predictor (odds ratio, 18.86; 95\% confidence interval, 1.59-223.22).

Conclusion: This study shows that a patient's willingness to quit is the most significant predictor of stopping smoking after Inpatient cessation Intervention for stroke Patients.

Keywords: Stroke; Inpatients; Smoking; Counseling; Smoking Cessation 


\section{INTRODUCTION}

Ever since the question was raised by Doll and Hill ${ }^{1)}$ in the 1940s of the association between lung cancer and smoking, numerous studies have been conducted into the association of smoking with lung cancer and chronic diseases. ${ }^{2)}$ Diseases associated with smoking include cancer, cardiovascular diseases, respiratory diseases, and reproduction-related diseases. Active research has also been conducted internationally. In 2008, a systematic study of Koreans reported that smoking was highly associated with lung cancer, gastric cancer, liver cancer, esophageal cancer, bladder cancer, cancer of the oral cavity, and cervical cancer. $^{2)}$

Smoking is the most significant factor associated with chronic disease and premature death. The three most important causes of death in Koreans in 2011 were malignant cancer, cerebrovascular disease, and cardiovascular disease, which together constituted $47.7 \%$ of total deaths. About $30 \%-40 \%$ of all deaths caused by cancer are attributed to smoking, and smoking is estimated to contribute approximately $30 \%$ to risk of death caused by cardiovascular disease, chronic obstructive pulmonary disease, and diabetes. ${ }^{2)}$ However, because treatments are available and smoking cessation itself can reduce the risk, smoking cessation is important.

Since the National Health Promotion Act was implemented in 2012, smoking is prohibited in all areas of medical institutions including outdoor resting spaces and parking structures. Patients hospitalized for any disease are instructed not to smoke within the hospital and have to be forced to quit smoking or be inconvenienced by going out of the hospital to smoke. Smoking in hospitals is also prohibited in the US by the policy of the Joint Commission. For hospitalized smokers, hospitalization itself is an opportunity to quit smoking. ${ }^{4)}$

Intensive counseling can be performed with hospitalized patients, and in a recent systematic study it was shown that such counseling increased the smoking cessation rate. ${ }^{5)}$ Furthermore, hospitalization becomes an opportunity to inform patients with tobacco-related diseases about the risks of smoking and to strengthen their motivation to quit smoking. ${ }^{4}$ Many studies of smoking cessation counseling have been conducted with patients hospitalized for smoking-related diseases, such as lung and cardiovascular diseases, and the results show a high cessation rate in this group. ${ }^{5)}$

While many studies of chronic and cardiovascular diseases have been conducted, studies of smoking cessation counseling for patients hospitalized with stroke are relatively limited. ${ }^{6}$ One domestic study analyzed the smoking cessation success rate among hospitalized patients who had received counseling. ${ }^{7)}$ While a previous study investigated whether stroke patients stopped smoking after discharge from the hospital, no study has investigated the factors affecting the success of smoking cessation in such patients after receiving counseling for smoking cessation. ${ }^{8)}$ As smoking is one of the risk factors for stroke, which has a high potential for relapse, we investigated the factors associated with the success of smoking cessation in stroke patients who received smoking cessation counseling.

\section{METHODS}

\section{Subjects}

The subjects of this study were stroke patients hospitalized in the neurology department of Konkuk University Medical Center in Seoul from March 1 to October 31, 2014. Smoking status was determined through the nursing information survey and interviews by attending physicians at admission. Male patients who smoked within 1 month of admission or were smoking at the time of admission were included.

Those who could not continue smoking cessation counseling due to complications of stroke (continued deterioration of consciousness, aphasia, cognitive impairment, etc.), who were so disabled that they could not smoke voluntarily, or whose modified Rankin Scale score was above 3 were excluded from the study. ${ }^{9}$ In addition, those who could not be contacted by telephone 3 months later or whose smoking cessation status could not be verified by the outpatient follow-up observation were also excluded from the study. A total of 39 patients were included in the study.

\section{Setting}

Stroke patients verified as smokers at admission were introduced to the smoking cessation counseling conducted by the hospital, and those who agreed to participate provided written consent. Counseling sessions were conducted after acute treatment for stroke was completed and when patients were in a stable condition, which was about 2-3 months after hospitalization. After performing a brain magnetic resonance imaging related to the stroke, only those patients diagnosed with ischemic stroke by the neurologist were included in the study. The disability status, National Institutes of Health Stroke Scale (NIHSS), and modified Rankin Scale were verified by the results of tests conducted by neurologists and residents during hospitalization., ${ }^{9,10)}$

Smoking cessation counseling was conducted with patients according to their identified smoking cessation behavioral change stage and questionnaire on smoking cessation status. Outpatient visits were encouraged for the patients for continuous cessation counseling after discharge, and the smoking cessation status was verified 3 months later in patients who were discharged after receiving smoking cessation counseling. Verification was made via telephone calls using the contact information recorded during hospitalization. Those who had stopped smoking were congratulated and encouraged to continue, whereas those who failed were asked to retry and receive counseling again.

Subjects were divided into two groups and compared: those who succeeded in smoking cessation after discharge (the quit-smoking group), and those who were still smoking (the still-smoking group). Age, gender, occupation, education level, and drinking level of the patients in both groups were collected through the admission record and nursing information survey. Current employment status was verified, and education level was divided into below middle school graduation, above high school graduation, and above college graduation. Weekly drinking amount was verified for the drinking level, and patients were 
divided into two groups based on whether drinking was above or below 14 servings per week.

\section{Intervention}

A doctor who was trained in smoking cessation counseling visited the ward and conducted the smoking cessation survey and counseling one-to-one. The smoking cessation questionnaire was composed of questions related to current daily smoking level, smoking duration, the Korean version of the Fagerstrom Test for Nicotine Dependence (FTND-K) scale, personal opinion of smoking cessation, confidence in the success of smoking cessation, history of past smoking cessation attempts and duration, status of withdrawal symptoms, situations in which one is prone to smoke, the "Why I Smoke" test to verify the smoking habit, reasons to quit smoking, and goal to achieve through smoking cessation. ${ }^{11)}$

The smoking cessation counseling with stroke patients was completed in about 10 minutes. Based on the smoking cessation preparation stage of patients, counseling was conducted by applying the $5 \mathrm{~A}$ and 5R. The 5A involves asking about the smoking status, advising on smoking, assessing smoking cessation intention, assisting smoking cessation, and arranging follow-up observation stages. To motivate smoking cessation in all patients participating in the smoking cessation counseling, these stages were applied to the entire counseling process. First, the smoking history was verified through visits to patients, and a survey was conducted to assess the smoking status explained above (ask). Based on the results, the relationship between the current disease and smoking, health risk, and the necessity of smoking cessation were explained, and smoking cessation was recommended (advise). The patient's commitment to quit smoking was verified (assess). Patients who wanted to quit smoking were educated about the STAR rules, which consist of setting a quit day and goal (set a quit day), informing others about the cessation intention (tell), predicting difficulties related to cessation and searching for solutions (anticipate), and removing tobacco-related items (remove). Information about nicotine replacement therapy and use of medication as well as related guides were provided (assist). Finally, the counseling was completed by arranging visits to the smoking cessation clinic to receive regular counseling (arrange).

For patients without commitment to quit smoking, the 5R principle was applied to induce smoking cessation motivation. The importance of smoking cessation was explained in relation to the personal situation of the patient (relevance), and the short- and long-term risks of continuing smoking were explained (risk). Patients were also informed of the benefits and advantages of smoking cessation (rewards), counseled on potential obstacles in the cessation process (roadblock), and repeatedly informed of the methods of smoking cessation counseling (repetition).12,13)

Although patients are in a non-smoking status once hospitalized due to the non-smoking policy, the smoking cessation behavioral change stage of patients was verified by directly asking whether they would continue to smoke. While the smoking cessation behavioral change stage was supposed to be divided into a pre-consideration stage, consideration stage, preparation stage, execution stage, and maintenance stage, there was an issue as to how to classify people who had already quit smoking after hospitalization but before counseling began. These people were considered to be at the execution-preparation stage or the willing-to-quit stage, and counseling for them was conducted according to the preparation stage. In addition, the group without clear commitment to quit smoking was classified as being at the pre-consideration stage.

Based on the FTND-K score, nicotine dependence was assessed and classified into mild ( $0-3$ points), moderate (4-6 points), and severe ( 7 10 points). Smoking cessation treatment through cognitive behavioral therapy (CBT) was recommended to the mild nicotine dependence group, whereas nicotine replacement therapy or medication was recommended to the other two groups. ${ }^{13,14)}$ However, nicotine replacement therapy or medication was prescribed only to those who wanted it after verifying the patient's opinions of the treatments.

\section{Statistical Analysis}

To identify the factors that can influence the success of smoking cessation after discharge, the t-test and chi-square test were performed comparing the still-smoking group with the quit-smoking group. In addition, multivariate logistic regression analysis was performed. The IBM SPSS Statistics for Windows software ver. 20.0 (IBM Co., Armonk, NY, USA) was used to process the data at the significance level of $95.0 \%$.

\section{RESULTS}

The number of patients referred for smoking cessation among the patients hospitalized for stroke from March to October of 2014 was 53. Of the 53, three female patients and four male patients who could not participate in counseling due to complications of stroke or maintaining smoking cessation voluntarily were excluded. In addition, a total of seven patients could not be contacted in the cessation status confirmation process after discharge. Therefore, a total of 39 patients were included in this study.

Of the 39 patients, 27 (69.2\%) were maintaining smoking cessation 3 months after discharge, whereas 12 (30.8\%) were smoking. The stillsmoking group after discharge and the quit-smoking group that succeeded in smoking cessation are compared in Table 1.

The average age of the quit-smoking group and the still-smoking group was $60.15 \pm 9.5$ years and $58.75 \pm 12.05$ years, respectively, with no significant difference between the two groups $(\mathrm{P}=0.368)$. There was also no difference in the average smoking amount (35.07 \pm 18.14 pack year vs. $36.92 \pm 18.27, \mathrm{P}=0.989$ ), weekly alcohol consumption level (17.37 \pm 19.20 units per week vs. $28.33 \pm 31.7, \mathrm{P}=0.103$ ), and NIHSS at admission ( $4.44 \pm 3.8$ vs. $4.0 \pm 2.59, \mathrm{P}=0.460$ ).

Furthermore, there was no significant difference between the two groups in employment status, nicotine dependence (FTND-K), past smoking cessation attempt status, and types of smoking cessation 
Table 1. Clinical characteristics of subjects $(N=39)$

\begin{tabular}{|c|c|c|c|}
\hline Characteristic & Still smoking $(n=12)$ & Quit smoking ( $\mathrm{n}=27$ ) & P-value* \\
\hline Age (y) & $58.75 \pm 12.05$ & $60.15 \pm 9.5$ & 0.37 \\
\hline Alcohol consumption (units per week) & $28.33 \pm 31.7$ & $17.37 \pm 19.20$ & 0.10 \\
\hline Duration of hospitalization (d) & $11.92 \pm 12.50$ & $17.04 \pm 18.7$ & 0.39 \\
\hline NIHSS at admission & $4.0 \pm 2.59$ & $4.44 \pm 3.8$ & 0.46 \\
\hline NIHSS at discharge & $3.0 \pm 2.98$ & $3.0 \pm 3.26$ & 0.55 \\
\hline Modified Rankin Scale at discharge & $2.0 \pm 1.65$ & $1.4 \pm 1.55$ & 0.72 \\
\hline Amount of smoking (pack year) & $36.92 \pm 18.27$ & $35.07 \pm 18.14$ & 0.99 \\
\hline \multicolumn{4}{|l|}{ Occupation } \\
\hline No & $4(26.7)$ & $11(73.3)$ & 0.73 \\
\hline Yes & $8(33.3)$ & $16(66.7)$ & \\
\hline \multicolumn{4}{|l|}{ Alcohol consumption (units/wk) } \\
\hline$\leq 14$ & $5(25.0)$ & $15(75.0)$ & 0.50 \\
\hline$>14$ & $7(36.8)$ & $12(63.2)$ & \\
\hline \multicolumn{4}{|l|}{ Education } \\
\hline Middle school & $6(50.0)$ & $8(29.6)$ & 0.45 \\
\hline High school & $3(25.0)$ & $11(40.7)$ & \\
\hline College & $3(25.0)$ & $8(29.6)$ & \\
\hline \multicolumn{4}{|l|}{ Cigarettes per day } \\
\hline$\leq 10$ & $2(33.3)$ & $4(66.7)$ & 0.74 \\
\hline $11-20$ & $4(44.4)$ & $5(55.6)$ & \\
\hline $21-30$ & $4(23.5)$ & $13(76.5)$ & \\
\hline$\geq 31$ & $2(28.6)$ & $5(71.4)$ & \\
\hline \multicolumn{4}{|l|}{ Time before smoking after waking } \\
\hline After 60 min & $2(66.7)$ & $1(33.3)$ & 0.36 \\
\hline 6 to $30 \mathrm{~min}$ & $1(14.3)$ & $6(85.7)$ & \\
\hline 31 to $60 \mathrm{~min}$ & $2(22.2)$ & $7(77.8)$ & \\
\hline Within 5 min & $7(35.0)$ & $13(65.0)$ & \\
\hline \multicolumn{4}{|c|}{ Difficulty refraining from smoking in places where it is forbidden } \\
\hline No & $6(24.0)$ & $19(76.0)$ & 0.28 \\
\hline Yes & $6(42.9)$ & $8(57.1)$ & \\
\hline \multicolumn{4}{|l|}{ Which cigarette would you most hate to give up? } \\
\hline Any other & $10(40.0)$ & $15(60.0)$ & 0.15 \\
\hline The first one in the morning & $2(14.3)$ & $12(85.7)$ & \\
\hline \multicolumn{4}{|c|}{ Smoke more frequently during the first hours after waking } \\
\hline No & $10(40.0)$ & $15(60.0)$ & 0.15 \\
\hline Yes & $2(14.3)$ & $12(85.7)$ & \\
\hline \multicolumn{4}{|c|}{ Smoke even when you are so ill that you are in bed for most of the day } \\
\hline No & $9(37.5)$ & $15(62.5)$ & 0.30 \\
\hline Yes & $3(20.0)$ & $12(80.0)$ & \\
\hline \multicolumn{4}{|l|}{ Stage of readiness to quit } \\
\hline Precontemplation & $5(83.3)$ & $1(16.7)$ & 0.03 \\
\hline Contemplation & 0 & 0 & \\
\hline Preparation & $2(10.5)$ & $17(89.5)$ & \\
\hline Action & $5(35.7)$ & $9(64.3)$ & \\
\hline \multicolumn{4}{|l|}{ Past attempt to quit } \\
\hline No & $7(36.8)$ & $12(63.2)$ & 0.50 \\
\hline Yes & $5(25.0)$ & $15(75.0)$ & \\
\hline \multicolumn{4}{|l|}{ Cessation treatment } \\
\hline Consult only & $9(40.9)$ & $13(59.1)$ & 0.31 \\
\hline Nicotine replacement therapy & $2(33.3)$ & $4(66.7)$ & \\
\hline Bupropion & 0 & 1 & \\
\hline Champix & $1(10.0)$ & $9(90.0)$ & \\
\hline Dyslipidemia & $1(8.3)$ & $5(3.7)$ & 0.645 \\
\hline Hypertension & $6(50.0)$ & $14(51.8)$ & 0.915 \\
\hline Diabetes mellitus & $8(66.7)$ & $11(40.7)$ & 0.176 \\
\hline
\end{tabular}

Values are presented as mean \pm standard deviation or number of subjects (\%).

NIHSS, National Institutes of Health Stroke Scale.

*From chi-square test for binary outcomes comparing the difference between the still-smoking group and quit-smoking group. 
Table 2. Multivariable analysis of smoking cessation factors*

\begin{tabular}{lccc}
\hline & Odds ratio & $\begin{array}{c}95 \% \text { confidence } \\
\text { interval }\end{array}$ & P-value \\
\hline Alcohol $^{\dagger}$ & 0.54 & $0.11-2.82$ & 0.46 \\
FTND-K score & 1.07 & $0.72-1.60$ & 0.73 \\
Past history of quitting smoking & 0.67 & $0.10-4.31$ & 0.67 \\
Use of treatment $^{\text {Readiness to quit }}{ }^{\dagger}$ & 2.10 & $0.33-13.36$ & 0.43 \\
\hline
\end{tabular}

FTND-K, Korean version of the Fagerstrom Test for Nicotine Dependence.

*Using a logistic regression model, adjusted for alcohol consumption, FTND-K, type of smoking cessation treatment, and stage of readiness to quit. ${ }^{\dagger}$ Alcohol consumption is grouped by under 14 glasses per week and over 14 glasses per week. *Readiness to quit is the precontemplation group (unwilling to quit) and preparation-action group (willing to quit).

therapies. However, when the two groups were compared, only the smoking cessation behavioral change stage of the patients showed a significant difference in success of smoking cessation.

Previous studies have proposed drinking history, nicotine dependence (FTND-K), past smoking cessation status, types of smoking cessation therapy, and smoking cessation behavioral change stage as the factors associated with success of smoking cessation. Multivariate logistic regression analysis was performed after correcting for the above variables, and a significant result was confirmed for the smoking cessation behavioral change stage. The odds ratio of patients in the preparation-execution stage was 18.86 times higher than that of patients in the pre-consideration stage (95\% confidence interval, 1.59-223.22) (Table 2).

\section{DISCUSSION}

This study was conducted to investigate the success of smoking cessation in adult male patients hospitalized for stroke and the factors associated with the success. The short-term success rate of smoking cessation at 3 months post discharge was $69.2 \%$ (27 out of 39 patients). There was a significant difference in the smoking cessation behavioral change stage at the time of counseling between the quit-smoking group and the still-smoking group.

There have been studies in other countries into smoking cessation counseling conducted with hospitalized stroke patients. In a study by Brunner et al., ${ }^{15)}$ when a follow-up observation was made 6 months after smoking cessation counseling was conducted in two groups, the smoking cessation success rate of the group that received 'high-intensity' counseling versus 'short' counseling was $42.9 \%$ and $37.8 \%$, respectively. In addition, in a study conducted by Sauerbeck et al., ${ }^{16)} 43 \%$ of stroke patients who were educated about the risks of smoking reportedly quit smoking when followed-up after 3 months.

The success rate of smoking cessation in this study (69.2\%) is higher than that in previous studies. While the study conducted by Brunner et al. ${ }^{15)}$ is similar to this study in that high-intensity counseling was conducted, it is difficult to make a direct comparison for several reasons. In the Brunner et al. ${ }^{15)}$ study, the observation was made for 6 months, the patients were younger (in their 20s and 30s), and included not only stroke patients but also transient ischemic attacks patients. ${ }^{15)}$ The study by Sauerbeck et al. ${ }^{16)}$ is different from the present study in that there was no follow-up by prescription of nicotine replacements or therapeutic drugs. ${ }^{16)}$

A study was published in 2011 of smoking cessation in hospitalized stroke patients in Korea. The results of a 1-year follow-up observation of 72 discharged patients diagnosed with stroke showed that $40.3 \%$ were still not smoking, and the past daily smoking amount before stroke diagnosis acted as an important factor. ${ }^{8)}$ However, there was no difference in the daily smoking amount between the two groups in this study (35.07 \pm 18.14 vs. $36.92 \pm 18.27, \mathrm{P}=0.989)$. The former study did not conduct smoking cessation counseling on stroke patients and only verified the cessation status after discharge. Compared to this study, where specific smoking cessation methods and prescriptions were provided during hospitalization, counseling during hospitalization appears to be more important than the daily smoking amount in maintaining smoking cessation.

This study identified the smoking cessation behavioral change stage as a significant factor affecting success of smoking cessation. A study by Ong et al ${ }^{17)}$ in 2015 identified nicotine dependence, level of motivation to quit smoking, and health status in the early hospitalization period as factors affecting success with smoking cessation. According to a study reported by Rigotti et al., ${ }^{18)}$ strengthening patient's motivation was more important than patient education for a short period when the long-term smoking cessation result was considered. Although the FTND-K and NIHSS scores did not show any significant difference for success of smoking cessation in this study, a similar result was shown in that the motivation to quit smoking corresponded to the preparation and active stages in the smoking cessation behavioral change stage.

A systematic study was published in 2012 about smoking cessation counseling for patients hospitalized for myocardial infarction and other related diseases. According to the results of that study, high-intensity counseling, with counseling over 10 minutes and follow-up observation within at least a month, was effective for successful smoking cessation, regardless of the patient's disease at admission. While adding nicotine replacement therapy along with counseling can increase the success rate of smoking cessation, there is not enough evidence for bupropion or varenicline. ${ }^{5)}$ In this study, the counseling of hospitalized patients was conducted for over 10 minutes and they were encouraged to visit the smoking cessation clinic after discharge. However, only nine (23\%) patients actually visited the smoking cessation clinic. Considering that the outpatient visit status was the choice of the patients themselves and most patients were receiving regular neurological care related to stroke, they seemed to have failed to recognize the importance of smoking cessation and that regular smoking cessation counseling is necessary for successful cessation.

A number of previous studies have demonstrated the effectiveness of CBT along with the use of smoking cessation drugs, and guidelines also recommend the use of drugs such as varenicline and bupropi- 
on. ${ }^{13,19)}$ However, in this study, $66.6 \%$ of the patients who attempted to quit smoking wanted only CBT rather than the use of smoking cessation drugs. Therefore, the number of subjects was too small to investigate whether the use of smoking cessation drugs was effective.

The significance of this study lies in the use of a systematic smoking cessation counseling method with domestic hospitalized stroke patients, and we attempted to identify factors influencing the maintenance of smoking cessation. However, there are limitations to this study. The number of patients (39) was too small to identify differences between the two groups, which limited the ability to obtain significant results other than what was found with regard to the patient's behavioral change stage. Furthermore, the verification of smoking cessation by self-report through telephone counseling was also a limitation. There is a possibility that some patients who reported to have quit smoking were in fact smoking, and that those who did not visit the hospital often and actively participate in care might have been missed by the telephone counseling. Thus, more patients who actively participated in care and smoking cessation treatment might have participated in telephone counseling.

Although this study was conducted with a small number of subjects, we confirmed a $69.2 \%$ rate of smoking cessation 3 months after highintensity counseling with hospitalized stroke patients. In addition, we identified the patient's behavioral change stage as the most important factor in smoking cessation. Hospitalization is an opportunity to inform smokers of the risks of smoking and to provide the motivation to quit smoking. Doctors should provide their patients with opportunities to receive counseling on smoking cessation and encourage them to quit smoking.

In that 3 months of smoking cessation is short term, future research should confirm whether the success of smoking cessation is maintained in the longer term. In addition, research needs to investigate how best to inform patients about the importance of regular smoking cessation counseling and about the methods that can ensure continuous counseling. Studies that consider the hospital visit schedule, frequency of follow-through from hospitalization to outpatient care, and how to provide proper motivation to hospitalized patients are needed. As the results of this study indicate, the smoking cessation preparation stage or motivation levels are important factors to consider and evaluate.

\section{CONFLICT OF INTEREST}

No potential conflict of interest relevant to this article was reported.

\section{REFERENCES}

1. Doll R, Hill AB. Smoking and carcinoma of the lung: preliminary report. Br Med J 1950;2:739-48.

2. Song HR, Kim CH. Epidemiology of the smoking-related diseases in
Korea. J Korean Acad Fam Med 2008;29:563-71.

3. National Center for Chronic Disease Prevention, Health Promotion Office on Smoking and Health. The health consequences of smoking: 50 years of progress: a report of the surgeon general. Atlanta (GA): Centers for Disease Control and Prevention; 2014.

4. Fiore MC, Goplerud E, Schroeder SA. The Joint Commission's new tobacco-cessation measures: will hospitals do the right thing? $\mathrm{N}$ Engl J Med 2012;366:1172-4.

5. Rigotti NA, Clair C, Munafo MR, Stead LF. Interventions for smoking cessation in hospitalised patients. Cochrane Database Syst Rev 2012;5: CD001837.

6. Edjoc RK, Reid RD, Sharma M. The effectiveness of smoking cessation interventions in smokers with cerebrovascular disease: a systematic review. BMJ Open [Internet]. 2012 [cited 28 February 2015];2:e002022. Available from: http://bmjopen.bmj.com/content/2/6/e002022.full. pdf+html.

7. Jung KY, Yoo SH, Ma SH, Hong SH, Lee YS, Shim UY, et al. Inpatient smoking cessation program and its success rate for abstinence among Korean smokers. Korean J Fam Med 2009;30:503-10.

8. Kang MS, Yoo SM, Kim JW, Kim KN, Lee SY, Kim JJ, et al. The change of smoking rates before and after hospital admission following diagnosis of ischemic stroke. JKSRNT 2011;2:97-103.

9. Van Swieten JC, Koudstaal PJ, Visser MC, Schouten HJ, van Gijn J. Interobserver agreement for the assessment of handicap in stroke patients. Stroke 1988;19:604-7.

10. National Institute of Neurological Disorders and Stroke. Stroke scale [Internet]. Bethesda (MD): National Institute of Health; 2003 [cited 2015 Feb 28]. Available from: http://www.ninds.nih.gov/doctors/ NIH_Stroke_Scale.pdf.

11. Ahn HK, Lee HJ, Jung DS, Lee SY, Kim SW, Kang JH. The reliability and validity of Korean version of Questionnaire for Nicotine Dependence. J Korean Acad Fam Med 2002;23:999-1008.

12. Park SW. Evidence-based smoking cessation counseling: motivational intervention and relapse prevention. J Korean Med Assoc 2011;54: 1036-46.

13. Fiore M. Treating tobacco use and dependence: 2008 update. Rockville (MD): US Department of Health and Human Services; 2008.

14. Raw M, McNeill A, West R. Smoking cessation: evidence based recommendations for the healthcare system. BMJ 1999;318:182-5.

15. Brunner Frandsen N, Sorensen M, Hyldahl TK, Henriksen RM, Bak S. Smoking cessation intervention after ischemic stroke or transient ischemic attack: a randomized controlled pilot trial. Nicotine Tob Res 2012;14:443-7.

16. Sauerbeck LR, Khoury JC, Woo D, Kissela BM, Moomaw CJ, Broderick JP. Smoking cessation after stroke: education and its effect on behavior. J Neurosci Nurs 2005;37:316-9, 325.

17. Ong KC, Cheong GN, Prabhakaran L, Earnest A. Predictors of success in smoking cessation among hospitalized patients. Respirology 2005; 10:63-9.

18. Rigotti NA, McKool KM, Shiffman S. Predictors of smoking cessation after coronary artery bypass graft surgery: results of a randomized trial with 5-year follow-up. Ann Intern Med 1994;120:287-93.

19. Noh HM, Paek YJ, Lee CM. Smoking cessation guidelines in the primary care setting. Korean J Fam Pract 2013;3:153-62. 